Bull. Austral. Math. Soc.

VOL. 50 (1994) [445-449]

\title{
ON PROJECTIVE LIFT AND ORBIT SPACES
}

\author{
T.K. DAS
}

\begin{abstract}
By constructing the projective lift of a $d p$-epimorphism, we find a covariant functor $\mathrm{E}$ from the category $\mathcal{C}_{d}$ of regular Hausdorff spaces and continuous $d p$ epimorphisms to its coreflective subcategory $\mathcal{E}_{d}$ consisting of projective objects of $\mathcal{C}_{d}$. We use $E$ to show that $E(X / G)$ is homeomorphic to $E X / G$ whenever $G$ is a properly discontinuous group of homeomorphisms of a locally compact Hausdorff space $X$ and $X / G$ is an object of $\mathcal{C}_{d}$.
\end{abstract}

\section{INTRODUCTION}

Throughout the paper all spaces are regular Hausdorff and maps are continuous epimorphisms. By $X, Y$ we denote spaces and for $A \subseteq X, \mathrm{Cl} A$ and Int $A$ mean the closure of $A$ and the interior of $A$ in $X$ respectively. The complete Boolean algebra of regular closed sets of a space $X$ is denoted by $R(X)$ and the Stone space of $R(X)$ by $S(R(X))$. The pair $\left(E X, h_{X}\right)$ is the projective cover of $X$, where $E X$ is the subspace of $S(R(X))$ having convergent ultrafilters as its members and $h_{X}$ is the natural map from $E X$ to $X$, sending $\mathcal{F}$ to its point of convergence $\cap \mathcal{F}$ (a singleton is identified with its member). We recall here that $\{\vartheta(F) \mid F \in R(X)\}$ is an open base for the topology on $E X$, where $\vartheta(F)=\{\mathcal{F} \in E X \mid F \in \mathcal{F}\}[8]$.

The projective lift of a map $f: X \rightarrow Y$ (if it exists) is the unique map $E f: E X \rightarrow$ $E Y$ satisfying $h_{Y} \circ E f=f \circ h_{X}$. In [4], Henriksen and Jerison showed that when $X$, $Y$ are compact spaces, then the projective lift $E f$ of $f$ exists if and only if $f$ satisfies the following condition, hereafter called the $H J$-condition, holds:

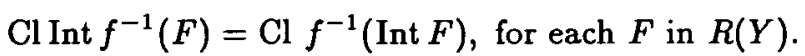

A condition for a map to satisfy equivalent to the $H J$-condition is that it is a $c$-map: a map $f: X \rightarrow Y$ satisfying $\mathrm{Cl}\left(f^{-1}(Y-F)\right)=X$ for all $F$ in $R(Y)$ [6,6G3]. Since the composition of $c$-maps need not be a $c$-map, we consider a special class of $c$-maps and obtain a category for our purpose.

Definition 1.1: A map $f: X \rightarrow Y$ is called a density preserving map (dp-map) if Int $\mathrm{Cl} f(A)$ remains nonempty whenever Int $A \neq \emptyset, A \subseteq X$.

Received 27 January 1994

Copyright Clearance Centre, Inc. Serial-fee code: 0004-9729/94 \$A2.00+0.00. 
A $d p$-map is a $c$-map. The converse need not be true. The composition of a $c$-map and a $d p$-map need not be a $c$-map. However, the composition of $d p$-maps is a $d p$-map.

In Section 2, we construct the projective lift of a $d p$-map. Denoting the category of regular Hausdorff spaces and continuous epimorphisms by $\mathcal{C}_{d}$ and by $\mathcal{E}_{d}$ its full subcategory having extremally disconnected spaces for objects we find that $\mathcal{E}_{d}$ is a coreflective subcategory of $\mathcal{C}_{d}$ and $E: \mathcal{C}_{d} \rightarrow \mathcal{E}_{d}$, sending $X$ to $E X$ and $f: X \rightarrow Y$ to $E f: E X \rightarrow E Y$ is a covariant functor.

Mioduszewski and Rudolf call a map $f: X \rightarrow Y$, where $X, Y$ are Hausdorff and $f$ not necessarily continuous, skeletal if $\operatorname{Int} f^{-1}(\mathrm{Cl} V) \subseteq \mathrm{Cl} f^{-1}(V)$ for each open set $V$ of $Y$ [5]. It may be noted that the $d p$-ness of a map is equivalent to it being a skeletal map. In [5] the authors do talk about the possibility of considering the functor $E$ when the morphisms are restricted to skeletal maps. However, neither any construction of the lift $E f$ is cited (we give one in Section 2) nor any property or application of the lift is studied like we do here in Section 3.

By inducing an action of a discrete group $G$ on $E X$, where $X$ is a $G$-space, Azad and Agrawal [1] showed that if $G$ is finite, then $E(X / G)$ is homeomorphic to the orbit space $E X / G$. An example to show that the above result may fail if $G$ is infinite, is provided in [1]. Here in Section 3, by showing the projective lift $E f$ of a $d p$-map $f$ from a locally compact space to a space to be an open map, we study the projective cover of an orbit space $X / G$, when $G$ is an infinite discrete group. We prove that if $G$ is a properly discontinuous group of homeomorphisms [7] of a locally compact Hausdorff space $X$, then $E(X / G)$ is homeomorphic to $E X / G$, whenever $X / G$ is an object of $\mathcal{C}_{d}$. For terms and notation not explained we refer to $[2,6,7,8]$.

\section{Projective Lift}

Lemma 2.1. Let $f: X \rightarrow Y$ be a dp-epimorphism. Then $\mathrm{Cl} f(F) \in R(Y)$ whenever $F \in R(X)$.

Proof: The result is immediate if $F=\emptyset$. Let $F \neq \emptyset$. Obviously ClInt $f(F) \subseteq$ Cl $f(F)$. For the reverse inclusion, we assume that $G \equiv Y-\mathrm{Cl} \operatorname{Int} \mathrm{Cl} f(F)$ intersects Cl $f(F)$. Then $\left[f^{-1}(G) \cap \operatorname{Int} F\right] \neq \emptyset$. Set $H=\mathrm{Cl}\left(f^{-1}(G) \cap \operatorname{Int} F\right)$. Since $f$ is a $d p$-map and $G$ is a regular open set we have $\emptyset \neq \operatorname{Int} C l f(H) \subseteq G \cap \operatorname{Int} \operatorname{Cl}(F)=\emptyset$, a contradiction.

Note 2.2: The following results follow from the above lemma:

(a) A closed $d p$-map is $R C$-preserving [3], that is, maps a regular closed set to a regular closed set.

(b) Let $f: X \rightarrow Y$ be a $d p$-epimorphism. Then for a regular closed set $H$ of $Y$ we have $\mathrm{Cl} f\left(\mathrm{Cl}^{-1}(\operatorname{Int} H)\right)=H$ and hence $R(Y)=\{\mathrm{Cl} f(F) \mid F \in$ $R(X)\}$. 
THEOREM 2.3. Let $f: X \rightarrow Y$ be a dp-epimorphism. Then the map $E f: E X \rightarrow$ $E Y$ sending $\mathcal{F} \in E X$ to the fixed ultrafilter $f_{\#}(\mathcal{F})=\{\mathrm{Cl} f(F) \mid F \in \mathcal{F}\}$, is the projective lift of $f$.

Proof: Let $H \in R(Y)$. If $H \cap \mathrm{Cl} f(F) \neq \emptyset$, for all $F \in \mathcal{F}$, then $\mathrm{Cl} f^{-1}$ (Int $\left.H\right) \in$ $\mathcal{F}$ and hence by note $2.2(\mathrm{~b}), H \in f_{\#}(\mathcal{F})$. Thus $f_{\#}(\mathcal{F}) \in S(R(Y))$. Further $f(\cap \mathcal{F})=$ $\cap f_{\#}(\mathcal{F})$ proves that $f_{\#}(\mathcal{F}) \in E Y$. Hence we have a map $E f: E X \rightarrow E Y$ defined by $E f(\mathcal{F})=f_{\#}(\mathcal{F})$. The commutativity of the following diagram establishes that $E f$ is an epimorphism:

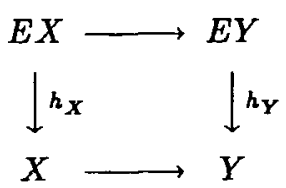

For continuity, one may observe that $E f\left(\vartheta\left(\mathrm{Cl} f^{-1}(\operatorname{Int} H)\right)\right) \subseteq \vartheta(H)$ for all $H$ in $R(Y)$. Finally, the uniqueness of $E f$ follows by recalling that a $d p$-map is a $c$-map [5,6G3].

Proposition 2.4. Let $\mathcal{C}_{d}$ be the category of regular Hausdorff spaces and continuous $d p$-epimorphisms and let $\mathcal{E}_{d}$ be the full subcategory of $\mathcal{C}_{d}$ having extremally disconnected spaces for objects. Then $\mathcal{E}_{d}$ is a coreflective subcategory of $\mathcal{C}_{d}$ such that $E: \mathcal{C}_{d} \rightarrow \mathcal{E}_{d}$ assigning $E X$ to $X$ and $E f: E X \rightarrow E Y$ to $f: X \rightarrow Y$ is a covariant functor.

Proof: By proving $\mathrm{Cl} E f(\vartheta(F))=\vartheta(\mathrm{Cl} f(F))$ for all $F$ in $R(X)$ we establish that the projective lift $E f$ of a $d p$-epimorphism $f$ is a $d p$-epimorphism. The rest of the proof is left to the reader.

REMARK 2.5. (a) A map $f: X \rightarrow Y$ is a $d p$-map if and only if the following stronger version of the $H J$-condition holds: for every $F$ in $R(Y), \operatorname{Cl} \operatorname{Int} f^{-1}(F)=$ $\mathrm{Cl} f^{-1}(d \operatorname{Int} F)$, where $d \operatorname{Int} F$ denotes an open set of $X$ which is dense in $F$.

(b) The class of non $d p$-maps satisfying the $H J$-condition consists precisely of the composition maps $f \circ g$, where $g$ is a $d p$-map and $f$ is a non $d p$-map satisfying the $H J$-condition.

\section{Projective cover of aN ORBIT SPACE}

Proposition 3.1. Let $f$ be a $d p$-map from a locally compact space $X$ to a space $Y$. Then the projective lift $E f$ is an open map.

Proof: It is sufficient to show that $E f(\vartheta(F))$ is open for all $F \in R(X)$. Choose $E f(\mathcal{F}) \in E f(\vartheta(F))$. Since $X$ is locally compact, there is a compact regular closed set $K$ in $X$ such that $K \subseteq F$ and $K \in \mathcal{F}$. By Lemma 2.1, $f(K) \in R(Y)$. We complete the proof by showing that $E f(\mathcal{F}) \in \vartheta(f(K)) \subseteq E f(\vartheta(F))$. Clearly $E f(\mathcal{F}) \in$ $\vartheta(f(K))$. Next we choose an $\mathcal{H}$ in $\vartheta(f(K))$ and observe that any finite subfamily of 
$\left.\mathcal{A} \equiv\{K\} \cup \mathrm{Cl} f^{-1}(\operatorname{Int} H) \mid H \in \mathcal{H}\right\}$ has nonempty meet. Moreover, compactness of $K$ guarantees that $\cap \mathcal{A} \neq \varphi$. Thus there is a $\mathcal{G}$ in $E X$ containing $\mathcal{A}$. Since $\mathcal{G}$ is a filter containing $K$ and $F$ contains $K$ so $\mathcal{G} \in \vartheta(F)$. Finally by maximality of $\mathcal{H}$, we get $E f(\mathcal{G})=\mathcal{H}$, this proves $\mathcal{H} \in E f(\vartheta(F))$.

REMARK 3.2. It is easy to show that the projective lift of the inclusion of the rationals into the reals is not open. This therefore justifies local compactness in the above proposition.

For a $G$-space $X$, the orbit $\{g \cdot x \mid g \in G\}$ of $x$ in $X$ is denoted by $G x$ and for $A \subseteq X$, the set $\{g . a \mid a \in A\}$, where $g \in G$, is denoted by $g . A$. Thus if $G$ is discrete, by defining $g . \mathcal{F} \equiv\{g \cdot F \mid F \in \mathcal{F}\}$ for an $\mathcal{F}$ in $E X$, one induces an action of $G$ on $E X$ [1]. We denote a member of $E(X / G)$ by $\mathcal{F}_{G x}$ and assume that the same converges to $G x$ in $X / G$.

THEOREM 3.3. Let $G$ be a properly discontinuous group of homeomorphisms on a locally compact Hausdorff space $X$ such that $X / G$ is Hausdorff. Then $E(X / G)$ is homeomorphic to $E X / G$.

Proof: Let $p: X \rightarrow X / G$ and $q: E X \rightarrow E X / G$ be the orbit maps. Since $p$ is an open map, it is a $d p$-map and hence the projective lift $E p$ of $p$ exists. Further, surjectivity of $E p$ is obvious and hence for $\mathcal{F}_{G_{x}} \in E(X / G)$ there exists an $\mathcal{F} \in E X$ such that $E p(\mathcal{F})=\mathcal{F}_{G x}$. By claiming that $E p^{-1}\left(\mathcal{F}_{G x}\right)=\{g . \mathcal{F} \mid g \in G\}$, the orbit $G \mathcal{F}$ of $\mathcal{F}$ in $E X$, we define a map $\eta: E(X / G) \rightarrow E X / G$ by $\eta\left(\mathcal{F}_{G x}\right)=q(\mathcal{F})$ and observe that $\eta$ is a homeomorphism. In fact, fibers of $E p$ being precisely the orbits gives us the bijectivity of $\eta$. The bicontinuity of $\eta$ is established by noting (i) openness of the maps $E p$ and $q$; and (ii) $\eta \circ E p=q$.

Proof of the CLAIM: $E p^{-1}\left(\mathcal{F}_{G x}\right)=\{g . \mathcal{F} \mid g \in G\}$.

From the definition of $E p$, we obtain $\{g . \mathcal{F} \mid g \in G\} \subseteq E q^{-1}\left(\mathcal{F}_{G x}\right)$. Let $\mathcal{H} \in$ $E p^{-1}\left(\mathcal{F}_{G x}\right)$ be such that $\mathcal{H} \neq g . \mathcal{F}$ for any $g$ in $G$. Then we complete the proof by obtaining a contradiction.

$E p(\mathcal{H})=\mathcal{F}_{G x} \Rightarrow \cap \mathcal{H} \in G x$. Since an element lies in a fibre of the map $E p$ if and only if the entire orbit determined by that element is contained in that fibre, we thus have $\{g \mathcal{H} \mid g \in G\} \subseteq E p^{-1}\left(\mathcal{F}_{G x}\right)$ and hence we can assume $\bigcap \mathcal{H}=x$. As $G$ is a properly discontinuous group of homeomorphisms on $X$, we can choose an open set $V$ containing $x$ such that $\{g . \mathrm{Cl} V \mid g \in G\}$ is a pairwise disjoint family of regular closed sets of $X$. Also, as $\mathcal{F} \neq \mathcal{H}$, there is a $K$ in $R(X)$ such that $K \in F$ and $K^{c} \in \mathcal{H}$. Now we set $K_{1}=K \wedge \mathrm{Cl} V$ and $K_{2}=K^{\mathrm{c}} \wedge \mathrm{Cl} V$ and observe (i) $K_{1} \in \mathcal{F}$ and $K_{2} \in \mathcal{H}$; and (ii) for all $g, h$ in $G, g . K_{1} \wedge h . k_{2}=\emptyset$. But this gives us invariant regular closed sets $H_{1} \equiv \bigvee_{g \in G} g . K_{1}$ and $H_{2} \equiv \bigvee_{g \in G} g . K_{2}$. Hence $p\left(H_{1}\right)$ and $p\left(H_{2}\right)$ are in $R(X / G)$. Also, $H_{1} \in \mathcal{F} ; H_{2} \in \mathcal{H}$ and $H_{1} \wedge H_{2}=\emptyset$. Finally, $p^{-1}\left(\operatorname{Int} p\left(H_{i}\right)\right)=\operatorname{Int} H_{i} ; i=1,2$ 
gives $p\left(H_{1}\right) \wedge p\left(H_{2}\right)=\emptyset$, and therefore $E p(\mathcal{H}) \neq E p(\mathcal{F})$. This contradicts the choice of $\mathcal{H}$.

Corlllary 3.4. Let $X$ and $G$ be as in Theorem 3.3. Then the projective cover of $X / G$ is $(E X / G, h)$, where $h: E X / G \rightarrow X / G$ is defined by $h(G \mathcal{F})=G(\cap \mathcal{F})$, the orbit in $X$ determined by $\cap \mathcal{F}$.

REMARK 3.5. Let $(X, d)$ be a locally compact metric space and let $G$ be a group of isometries acting on $X$ such that there exists a $\delta>0$ satisfying $d(g . x, h . x)>\delta$ for all $g, h$ in $G, g \neq h$ and $x \in X$. Then $X / G$ is metrisable and by Theorem 3.3, $E(X / G)$ is homeomorphic to $E X / G$.

EXAMPLE 3.6. From the above Remark it follows that for an $n$-torus $T^{n}, E\left(T^{n}\right)$ is homeomorphic to $E\left(\mathbb{R}^{n}\right) \mathbb{Z}^{n}$.

\section{REFERENCES}

[1] K.K. Azad and Gunjan Agrawal, 'On the projective cover of an orbit space', J. Austral. Math. Soc. Ser. A 46 (1989), 308-312.

[2] G.E. Bredon, Introduction to compact transformation groups (Academic Press, New York, 1972).

[3] T.K. Das, 'A note on maps preserving regular closed sets', Indian J. Math. (to appear).

[4] M. Henriksen and M. Jerison, 'Minimal projective extension of compact spaces', Duke Math. J. 32 (1965), 291-295.

[5] J. Mioduszewski and L. Rudolf, ' $H$-closed and extremally disconnected spaces', Dissertationes Math. 66 (1969), 1-55.

[6] J.R. Porter and R.G. Woods, Extensions and absolutes of Hausdorff spaces (Springer-Verlag, Berlin, Heidelberg, New York, 1988).

[7] R.C. Walker, The Stone Cech compactifaction (Springer-Verlag, Berlin, Heidelberg, New York, 1974).

[8] R.G. Woods, 'A survey of absolutes of topological spaces', in Topological Structures II, Centre Tracts 116 (Math. Centrum, Amsterdam, 1979), pp. 323-362.

Department of Mathematics

Faculty of Science

The MS University of Baroda

Vadodara 390002

India 